\title{
QUANTITATIVE ASSESSMENT OF CARTILAGE SURFACE ROUGHNESS IN OSTEOARTHRITIS USING HIGH FREQUENCY ULTRASOUND
}

\author{
Ronald S. Adler, ${ }^{\dagger}$ Dale K. Dedrick, ${ }^{\ddagger}$ Timothy J. Laing, ${ }^{*}$ \\ Edward H. Chiang, ${ }^{\dagger}$ Charles R. Meyer, ${ }^{\dagger}$ Peyton H. Bland ${ }^{\dagger}$ \\ and JONATHAN M. RUBIN ${ }^{\dagger}$
}

University of Michigan Medical Center, Department of Radiology, ${ }^{\dagger}$ Orthopaedic Research Laboratories, Orthopaedic Surgery and Department of Internal Medicine, Rheumatology Division, ${ }^{\ddagger}$ Department of Internal Medicine, Rheumatology Division, ${ }^{*} 1500$ E. Medical Center Drive, Ann Arbor, MI 49109/0030, USA

(Received 19 June 1991; in final form 17 September 1991)

\begin{abstract}
Osteoarthritis (OA) is a common disease which affects nearly $50 \%$ of people over age 60 . Histologic evaluation suggests that fibrillations $\sim 20-150 \mu \mathrm{m}$ are among the earliest changes in the articular cartilage. We propose a technique to quantify these surface fibrillatory changes in osteoarthritic articular cartilage by considering the angular distribution of the envelope-detected backscattered pressure field from an incident 30-MHz focused transducer. The angular distribution of the scattered acoustic field from an insonifying source will directly relate to the distribution of surface fibrillatory changes. Data are presented for three different grades $\mathbf{( 4 0 0 , 5 0 0}$ and 600 grit) of commercially available emory paper and three samples of osteoarthritic femoral head articular cartilage, which were visually assessed as having smooth, intermediate and rough surfaces, respectively. Our preliminary results indicate a probable monotonic relationship between articular cartilage roughening and the degree of broadening in the angle-dependent pressure amplitude. When applied to the emory paper, the technique indicates sensitivity to differences as small as $\sim 5-10 \mu \mathrm{m}$ in mean roughness. This procedure may provide an extremely sensitive and reproducible means of quantifying and following the cartilage changes observed in early osteoarthritis.
\end{abstract}

Key Words: Acoustics, Ultrasonics, Ultrasonic backscatter, Articular cartilage roughness, Osteoarthritis.

\section{INTRODUCTION}

Osteoarthritis $(\mathrm{OA})$ is a very common disease and the early morphologic and biochemical changes in the articular cartilage are well known. However, the initiating factors and exact sequence of events involving the subchondral bone and synovium remains conjectural (Mankin and Lippiello 1970; Minns et al. 1977; Bland and Cooper 1984). At present, treatment is limited to relatively advanced disease in part because of the inability to detect and monitor OA in its early presymptomatic stages. The development of a noninvasive technique to identify and follow early OA has important implications for assessing therapeutic regimens advocated for the treatment of the arthritis and

Address correspondence to: Ronald S. Adler, M.D., Ph.D., University of Michigan Medical Center, Dept. of Radiology, 1500 E. Medical Center Drive, Ann Arbor, MI 48109/0030.

Portions of this research supported in part by NIH Grant \#'s K11-AR01793 and 5P60-20557. the prevention of disability (Paulus 1989; Brandt 1990).

Histologic evaluation (Minns et al. 1977; Bland and Cooper 1984) suggests that fibrillations $\sim 20-150$ $\mu \mathrm{m}$ in height are among the earliest changes in the articular cartilage. These distance scales are particularly conducive to quantification by relatively highfrequency acoustic scattering $(10-30 \mathrm{MHz})$, which is within the range of commercially available transducers. In particular, the angular distribution of scattered acoustic radiation from some insonifying source will directly relate to variations in surface height, or roughness, resulting from the distribution of surface fibrillatory changes. In the simplest case, in which the surface fibrillations are sufficiently small relative to the acoustic wavelength, the angular scattering is a direct measure of surface root-mean-square (RMS) roughness (Thorne and Pace 1984). Similar surface phenomena have been extensively investigated within the nondestructive testing and laser speckle literature 


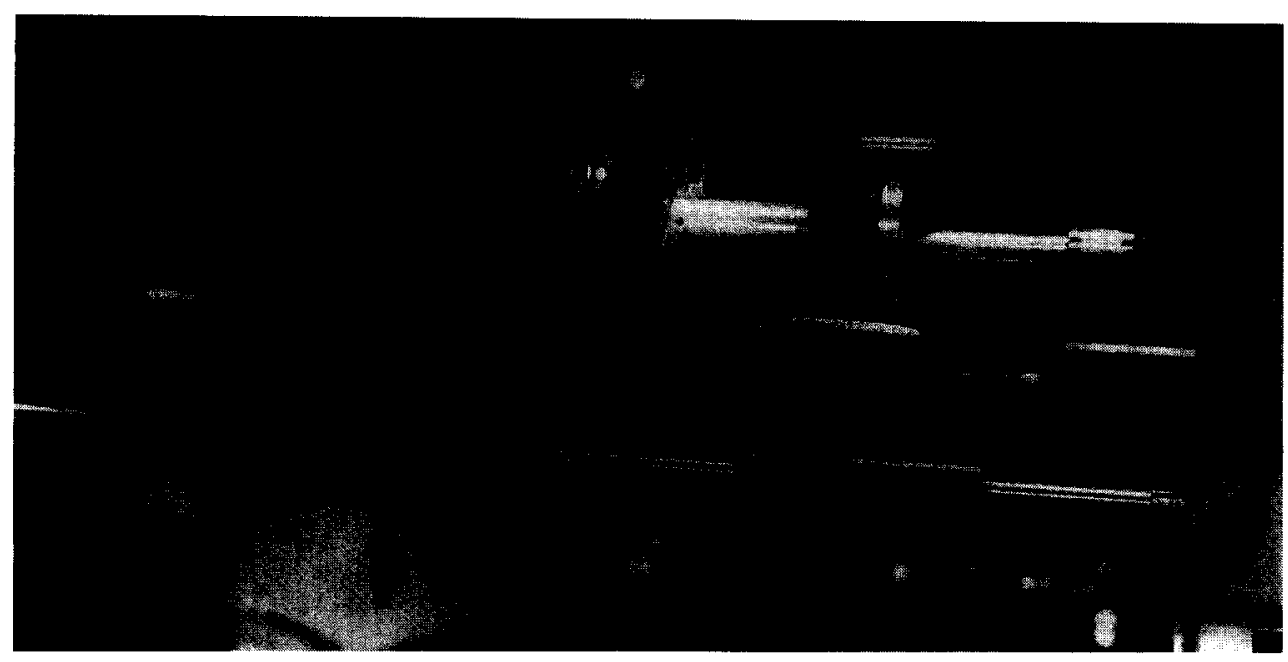

(a)

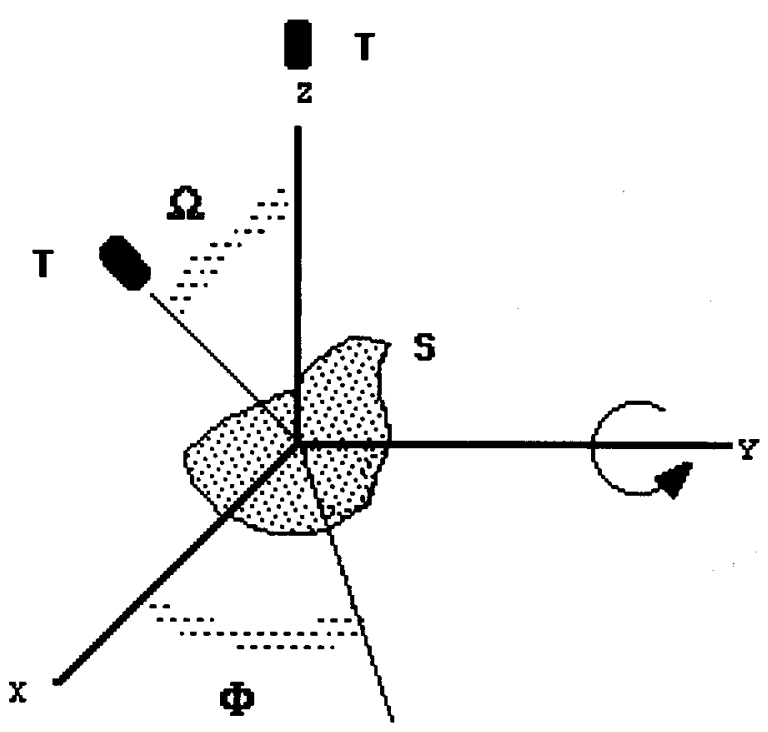

(b)
SRL 1712B CUI Block Diagram

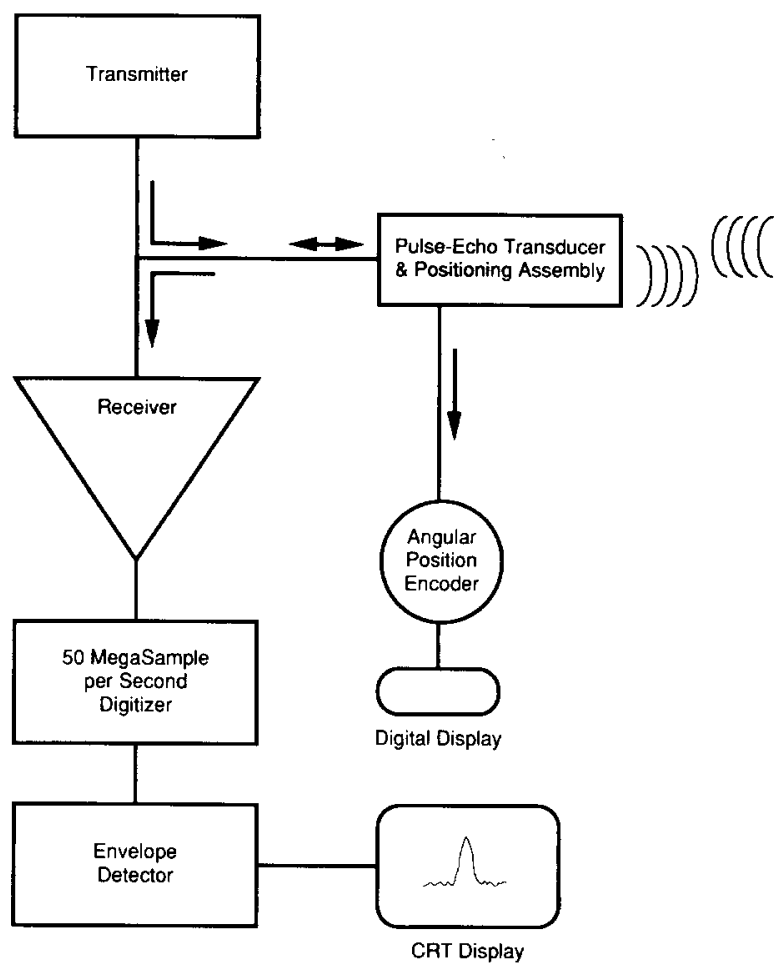

(c)

Fig. 1. (a) Photograph of scan apparatus used for ultrasound data acquisition (see description in text). (b) scan geometry. The interrogation angle $\Omega$ of the transducer T is determined by the degree of rotation about the $y$-axis. $\Phi$ denotes the azimuthal orientation of the sample, S. (c) Block schematic of ultrasonic instrumentation (SRL) for RF data acquisition, digitization and storage (see text).

(Quentin et al. 1975; De Billy et al. 1976; Thorne and Pace 1984; Goodman 1985).

We propose a technique to quantify surface fibrillatory changes in osteoarthritic articular cartilage which considers the angular distribution of the enve- lope detected backscattered pressure field from an incident $30-\mathrm{MHz}$ focused transducer. The measured acoustic field reflects the degree of surface roughening by the departure of the angular distribution from that expected from a specular reflector (i.e., perfectly 


\section{Data from "A600 emory"}

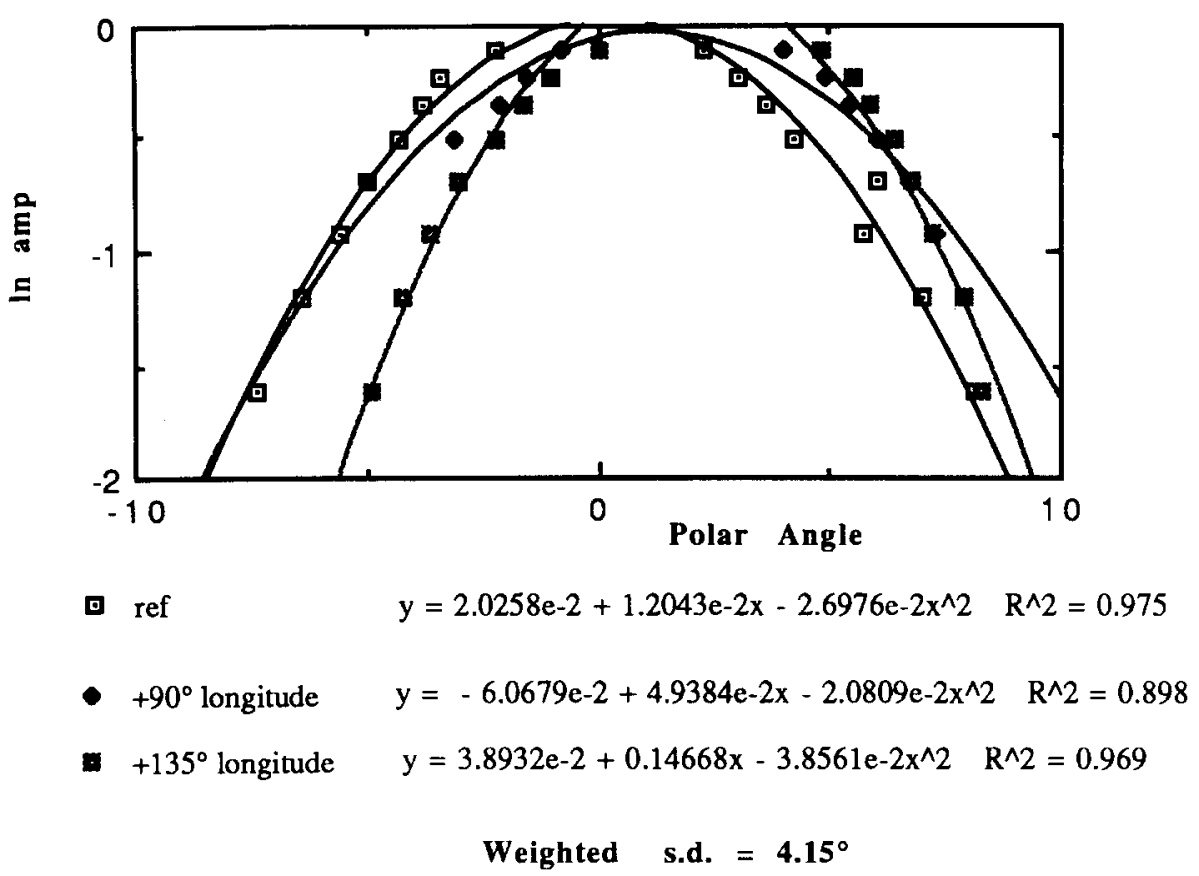

Fig. 2. Plot of In RF amplitude data taken from 600 grit size emory paper as a function of polar angle $\Omega$ for three different azimuthal orientations $\left(0^{\circ}, 90^{\circ}, 135^{\circ}\right)$. Quadratic regression curves are illustrated along with weighted angular SD. The latter are obtained from the coefficient of $x^{2}$ with weights determined by the $R^{2}$ for each fit.

$$
\operatorname{Ref}=0^{\circ} \text {. }
$$

smooth surface). Data are presented for three different grades (400, 500 and 600 grit) of commercially available emory paper and three samples of OA involved femoral head articular cartilage, which were visually assessed as having smooth, intermediate and rough surfaces, respectively. Our preliminary results indicate a probable monotonic relationship between articular cartilage roughening and the measured acoustic angular dispersion. Furthermore, the technique may allow one to detect differences in mean roughness as small as $\sim 5-10 \mu \mathrm{m}$, and if duplicated in vivo provide an extremely sensitive and reproducible means of quantifying and following the cartilage changes observed in early osteoarthritis.

\section{METHODS AND MATERIALS}

A photograph of the scan apparatus, simplified schematic of the scan geometry and block diagram of the ultrasonic instrumentation are depicted in Fig. 1. The transducer and sample are denoted by $T$ and $S$, respectively. The sample is placed onto a circular platform which may be positioned vertically and with respect to the azimuthal coordinate $\phi$. A $30-\mathrm{MHz}, 1$ $\mathrm{mm}$ active diameter ultrasonic transducer (Ultran Laboratories Inc., State College, PA) focused at $3 \mathrm{~mm}$
(Meyer et al. 1988a,b) is positioned within a 2.5-mm diameter hole in the distal position of an aluminum C-arm, Fig. 1b, the proximal portion of which is rigidly fixed to an electronically calibrated, manually operated rotor. The latter defines the axis of rotation of the transducer ( $y$-axis). The transducer is further positioned into a normal saline bath which surrounds the sample to a level several millimeters above the sample surface, corresponding to the focal zone of the transducer. The scan angle $\Omega$ is determined by the angular position of the $\mathrm{C}$-arm. In order to avoid differential attenuation and variable transducer effects, the interrogated surface is placed at the approximate center of rotation.

Table 1. Angular distribution data from emory paper.

\begin{tabular}{ccc}
\hline Grit size† & Median particle size & $\begin{array}{c}\text { SD } \\
\text { (degrees) }\end{array}$ \\
\hline 600 & $14.5 \mu \mathrm{m}$ & 4.15 \\
500 & $18.2 \mu \mathrm{m}$ & 4.77 \\
400 & $22.1 \mu \mathrm{m}$ & 5.59 \\
\hline
\end{tabular}

$\dagger$ Increasing grade refers to smaller mean grit size.

$\neq$ Calculated angular standard deviation assuming Gaussian angular distribution with weighted average performed over three different azimuthal orientations. 


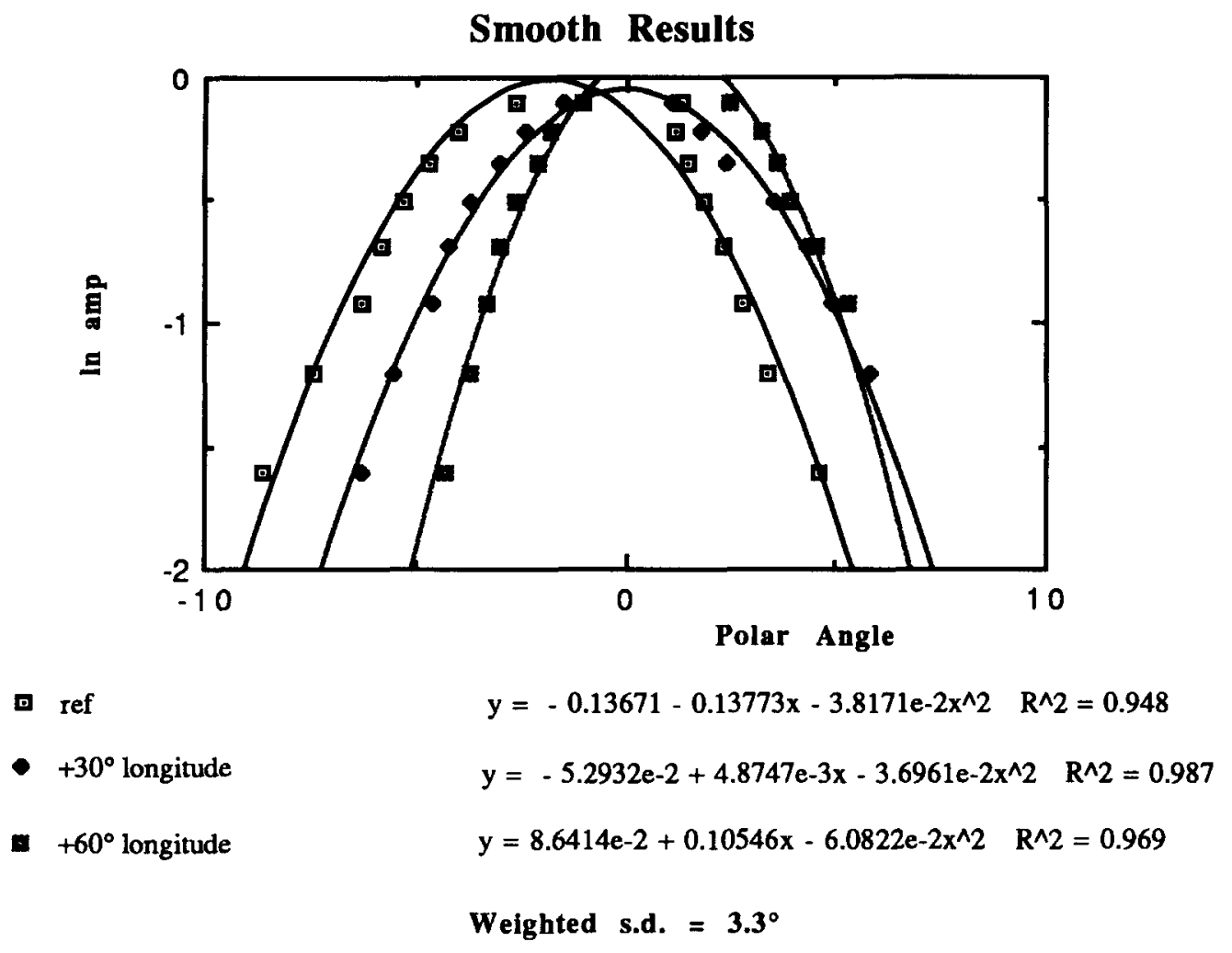

(a)

Intermed Results

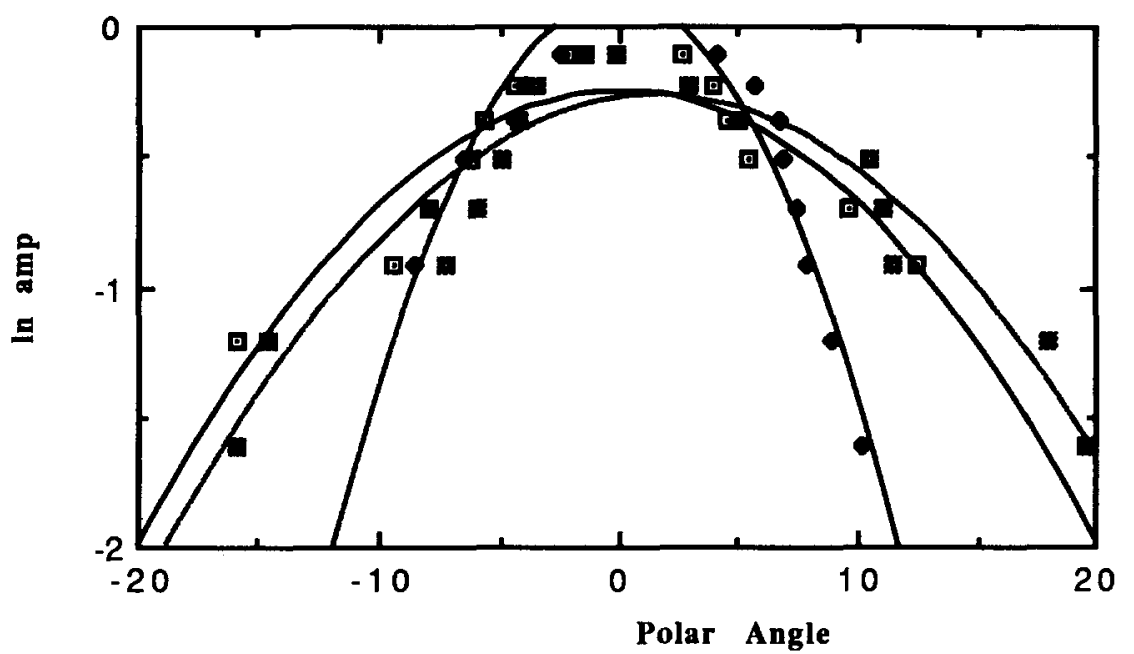

व ref

$y=-0.25039+6.4829 e-4 x-4.3296 e-3 x^{\wedge} 2 \quad R^{\wedge} 2=0.830$

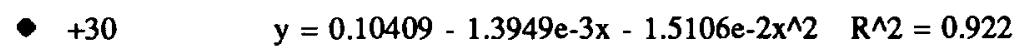

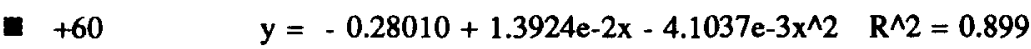

Weighted s.d. $=7.9^{\circ}$

(b)

Fig. 3. Plots of In RF amplitude data taken from the visually graded articular cartilage samples as a function of polar angle $\Omega$ for three different azimuthal orientations $\left(0^{\circ}, 30^{\circ}, 60^{\circ}\right)$. Quadratic regression curves are illustrated along with weighted angular SD: (a) smooth, (b) intermediate, (c) rough. The progressive broadening of these curves with increasing roughness is evident. Ref $=0^{\circ}$. No definable Gaussian fit is obtained in the rough sample for which individual nicks are resolved at $30 \mathrm{MHz}$. 


\section{Rough Results}

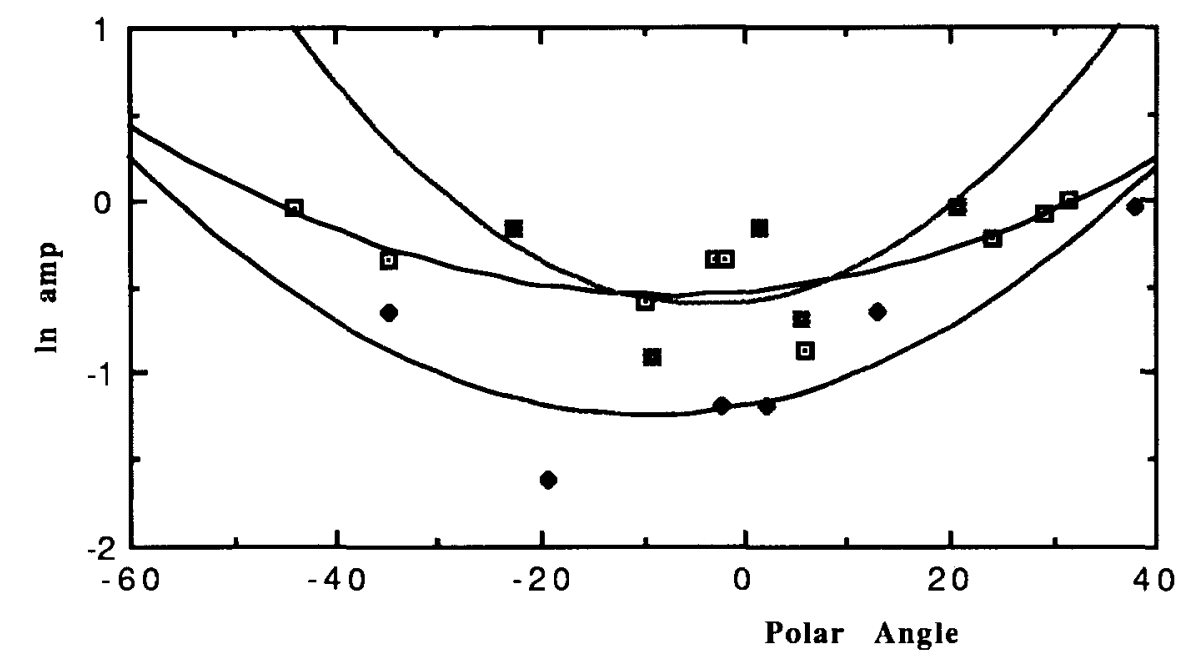

$$
\begin{array}{lll}
\text { ref } & y=-0.54493+4.9597 e-3 x+3.5326 e-4 x^{\wedge} 2 & R^{\wedge} 2=0.647 \\
+30 & y=-1.2045+1.1046 e-2 x+5.8902 e-4 x^{\wedge} 2 & R^{\wedge} 2=0.791 \\
-60 & y=-0.60442+7.6012 e-3 x+9.9613 e-4 x^{\wedge} 2 & R^{\wedge} 2=0.439
\end{array}
$$

(c)

Fig. 3. Continued.

The 30-MHz transducer has a $-6-\mathrm{dB}$ bandwidth of $12 \mathrm{MHz}$ with axial and lateral resolution of 100 and $150 \mu \mathrm{m}$ at the focus, respectively. The supporting ultrasonic instrumentation (Systems Research Laboratories, Inc., Dayton, OH), Fig. 1c, is employed as a pulser, linear amplifier, digitizer, B-mode display and data storage system. The pulse width of the pulser is optimized for the center frequency of the transducer. The receiver has a flat frequency response up to 30 $\mathrm{MHz}$. The 8-bit digitizer operates at a sampling frequency of $50 \mathrm{MHz}$. Since the folding frequency for this system is $25 \mathrm{MHz}$, radio frequency (RF) signal components at $30 \mathrm{MHz}$ are aliased down to $20 \mathrm{MHz}$. Estimated angular resolution $(\Delta \Omega)$ is $\sim 0.75^{\circ}$. The look angle is sensed by an optical encoder mounted on the transducer subsystem. The acquired RF is additionally stored on 3.5-in floppy disks for future reference (Meyer et al. 1988a,b).

Three different grades (400, 500 and 600 grit) of commercially available emory paper (3M Company, Tri-Mite, Minneapolis, MN) were first examined. While considerable overlap in grit size does occur in each of these, the distribution of particle size results in a smaller mean value with increasing grade. The corresponding grit size ranges of the study samples were $10.5-40.0,8.0-34.6$ and approximately 5.0$32.0 \mu \mathrm{m}$ with associated median sizes of $22.1,18.2$ and $14.5 \mu \mathrm{m}$, respectively (Grinding Wheel Institute, 1977). For the second set of observations, human cartilage samples from the femoral head articular surface were obtained from patients with OA undergoing hip replacement. On gross examination of the cartilage surface, these were divided into three categories: smooth, intermediate and rough. Samples of each were first sectioned into approximately $1-\mathrm{cm}^{3}$ rectangular plugs containing portions of the subchondral bone. Each plug was oriented with the cartilage surface toward the transducer. The free bone surface was affixed to a cylindrical cap (Fig. 1a) which was subsequently filled with normal saline covering both the specimen and active surface of the transducer. Following ultrasound data acquisition, histologic preparations of the cartilage specimens were obtained after standard paraffin embedding, sectioning and staining with hematoxylin and eosin as well as Safranin O. Light microscopic images of the cartilage surfaces magnified $10 \times$ were then digitized using a video camera linked to a VICOM 1850 image processing system (VICOM Systems Inc., San Jose, CA) to a $512 \times 512$ matrix with approximately 8 bit dynamic range. A series of morphologic operations were then applied to the resulting digitized image of the chondral surface in order to establish quantitative estimates of the extent of the fibrillatory changes. Distance calibration was 
accomplished by obtaining a digitized image of the intermediate roughness sample along with a transparent $1-\mathrm{cm}$ graticule containing millimeter divisions under otherwise identical conditions to those applied to the initially digitized cartilage histologic samples. The distances (in pixels) between common fiducial points on the cartilage surface of the two digitized intermediate roughness images (i.e., with and without the graticule) were used to verify this equivalence.

The digitized images were first visually thresholded producing a binary image depicting the cartilage contour. This was followed by interior pixel fill, logical shift and interior pixel remove operations resulting in a one-pixel thick estimate of the cartilage boundary which was subsequently superimposed on the initial digitized image to confirm accuracy of the approximated margin. A series of morphological operations (Serra 1986; Sternberg 1986; Zhuang and Haralick 1986) including fattening (32 iterations), hulling (40 iterations) and thinning (64 iterations) were then applied to this "rough" edge estimate, respectively, to obtain the low spatial frequency surface trend. The first two of these resulted in a gross surface trend with removal of high spatial frequency cusps by virtue of the hulling operation. Thinning applied to this gross surface trend produced a one-pixel thick skeleton curve from which the histologic mean surface roughness could be derived (see below).

\section{RESULTS}

Data from scans performed on emory paper and representative cartilage specimens from each category were displayed as plots of $\ln$ RF amplitude as a function of polar angle $\Omega$, for three different azimuthal orientations. The data were fit to Gaussian distributions (or equivalently, quadratic fits to $\log$-data). The standard deviation (SD) of each generated curve gives a measure of angular distribution. A weighted average of individual values of the quadratic coefficient was obtained with weights determined by $R^{2}$ values for each fit from which the weighted SD is obtained. A typical set of ln RF amplitude data for the 600 grade emory paper is given in Fig. 2. The $R^{2}$ for these data range from approximately .9 to .98 , indicating the validity of the Gaussian fit in this angular range. Similar results are present with the remaining emory paper samples. The results of the emory paper scans are presented in Table 1, indicating grade, median particle size and mean standard deviation. A trend toward larger SD values with increasing grit size is demonstrated.

Angular data for the three different cartilage specimens are presented in Fig. 3 and summarized in Table 2. Parabolic fits to the $\ln \mathrm{RF}$ data were poor $\left(R^{2}\right.$
Table 2. Angular distribution data from cartilage samples.

\begin{tabular}{lcc}
\hline Visual grade & Mean roughness $\dagger$ & $\begin{array}{c}\text { SD } \\
\text { (degrees) }\end{array}$ \\
\hline Smooth & $7.9 \mu \mathrm{m}$ & 3.3 \\
Intermediate & $29.1 \S \mu \mathrm{m}$ & 7.8 \\
Rough & $49.1 \mu \mathrm{m}$ & $* * *^{*}$ \\
\hline
\end{tabular}

$\uparrow$ Based on boundary estimates from digitized histologic images. ¥ Calculated angular standard deviation assuming Gaussian angular distribution with weighted average performed over three different azimuthal orientations.

* No definable Gaussian angular distribution. Individual nicks resolved at $30 \mathrm{MHz}$.

$\S$ Arithmetic average of two separate determinations (see text).

$=0.44-0.79$ ) in the "rough" case in which individual nicks are resolved in the reconstructed B-mode images. The resulting fit in this latter situation is close to being flat or slightly concave. This result is not surprising since at 30-MHz individual nicks are comparable in size to the acoustic wavelength. The SD values between smooth and intermediate articular cartilage samples differ by approximately a factor of two. In Table 2, the scan data are compared to absolute mean roughness based on the digitized cartilage images. The calculated area between approximated and smoothed (low spatial frequency) cartilage surfaces normalized to the smoothed surface arc length provides a mean roughness modulus estimate. Two separate determinations were obtained in the case of the intermediate roughness sample to crudely evaluate reproducibility which differed by approximately $1 \mu \mathrm{m}(28.6,29.7$ $\mu \mathrm{m})$. Examples of the estimated cartilage boundaries, low spatial frequency surface trends and corresponding histologic images are presented in Fig. 4. The rough edge estimates appear as black boundaries on the composite images formed with their associated cartilage histology.

\section{DISCUSSION}

Our preliminary work demonstrates that acoustic scattering is well suited to investigate surface roughness which are secondary to cartilage surface fibrillations occurring in OA. Areas of cartilage that differ in mean surface roughness by 20-30 $\mu \mathrm{m}$ are easily distinguished at $30 \mathrm{MHz}$. Concommitant changes in the cartilage itself include alterations of the collagen matrix, which may significantly affect the internal acoustic properties of the cartilage, changes in the state of hydration and diminished proteoglycan content (Mankin and Lippiello 1970; Minns et al. 1977; Bland and Cooper 1984). Changes in the surface impedance expected to occur with alterations in the cartilage matrix, would more likely affect the mag- 

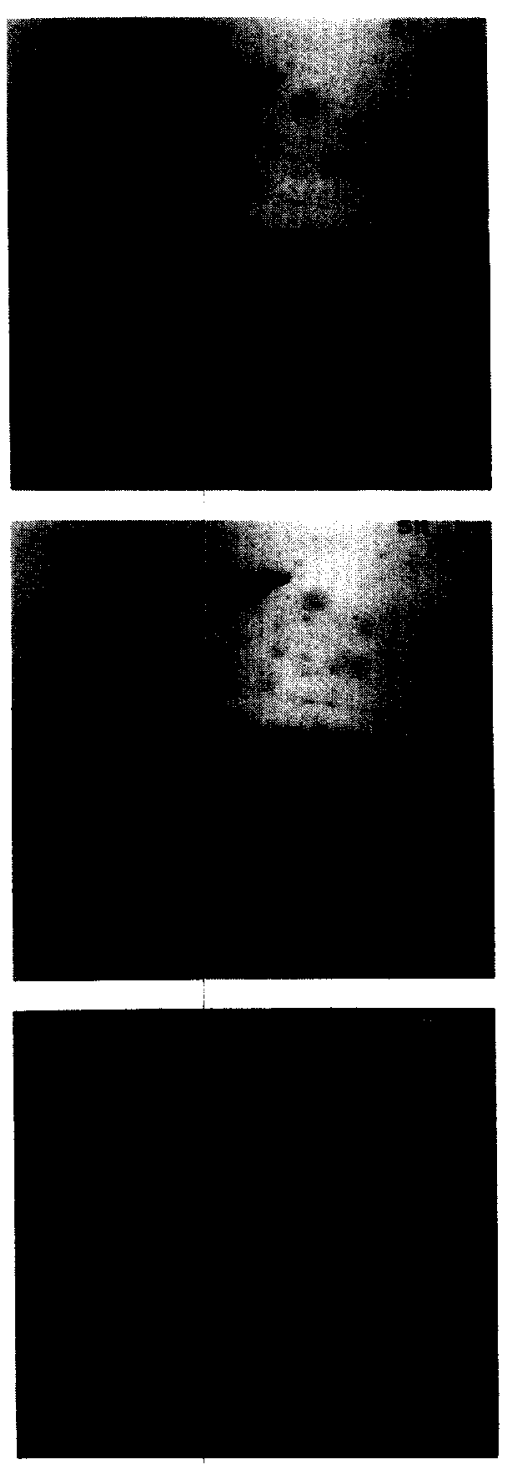

(a)
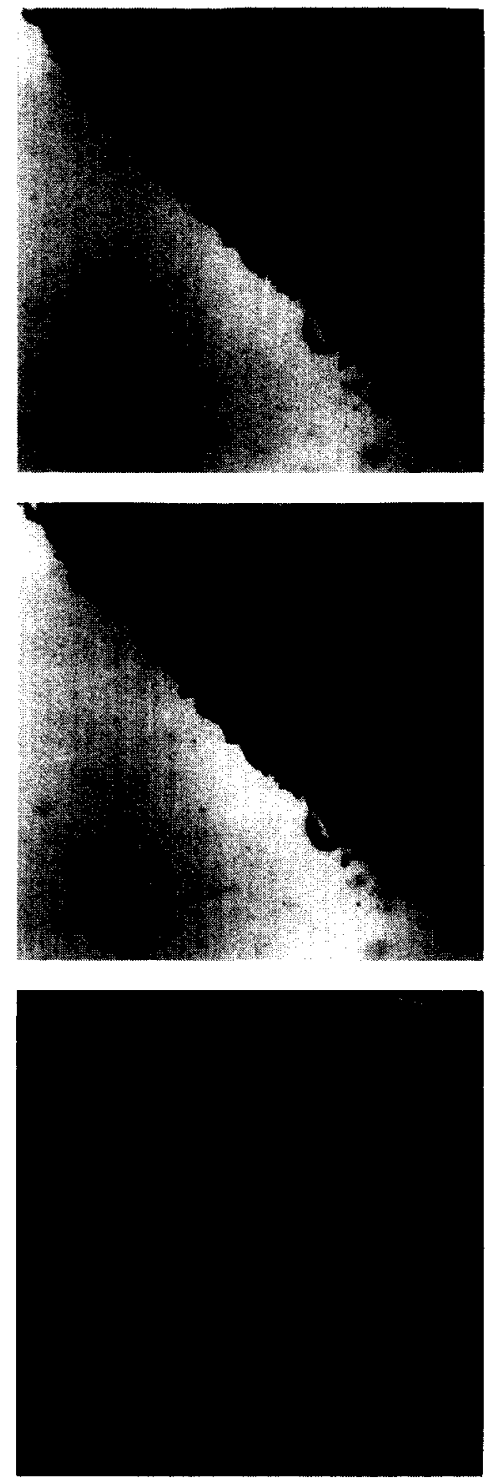

(b)
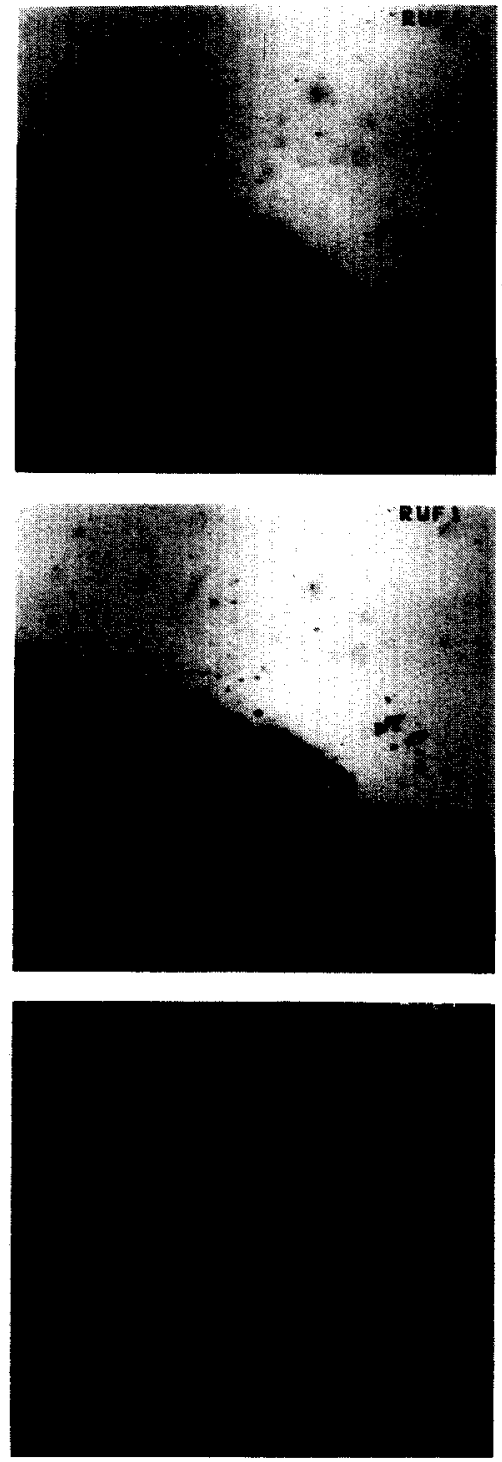

(c)

Fig. 4. Composite images of cartilage histology, estimated boundaries based on thresholding of VICOM image, and the low spatial frequency surface trend obtained from fattening, hulling and thinning operations for smooth (a), intermediate (b), and rough (c) articular cartilage samples. In each composite figure the top image depicts the VICOM image of the digitized histologic slide alone. The middle images contains a superimposition of the histology and estimated histologic boundary (black). The bottom image is a superimposition of the estimated histologic boundary and the low spatial frequency surface trend form which the absolute mean roughness may be calculated.

nitude of the backscattered pressure amplitude rather than the angular distribution. It is interesting to note that the concentration of collagen as well as the organization of the collagen matrix have the most significant effect on the acoustic attenuation and speed-of-sound measurements relative to the remaining constituents in the transmission experiments performed at 100 MHz by Agemura et al. (1990). A large sample of cartilage specimens in which both the angle-depen- dent acoustic backscatter and biochemical composition have been determined would be required to fully investigate the effects of scattering due to local impedance changes.

The averaging performed over multiple azimuthal directions enables several realizations of scattering from the same surface, but presupposes that there is symmetry about the axis of rotation. If indeed there is a preferred direction for fibrillations to occur, 
the mean surface roughness may display local anisotropy. Acoustic scattering off of roughened surfaces has been the subject of extensive investigation in the nondestructive testing and laser speckle literature (Quentin et al. 1975; De Billy et al. 1976; Thorne and Pace 1984; Goodman 1985), which in the simplest case relates the angular distribution of scattering to the RMS roughness. In a more realistic approximation (Goodman 1985), the angular distribution directly relates to the pair correlation function of the local height distribution $h(u, v)$,

$$
\Phi(x, y)=\langle h(u+x, v+y) h(u, v)\rangle-\langle h\rangle^{2},
$$

in which $(x, y)$ and $(u, v)$ denote surface points, and \langle\rangle indicates ensemble average over the distribution of the stochastic variable $h(u, v)$, taken to be stationary random process. The presence of localized angular correlations could then, in principal, be inferred.

The paucity of data do not permit an extended analysis but they do support the following two conclusions. First, the emory paper experiment demonstrates the ability of the current technique combined with a $30-\mathrm{MHz}$ transducer to discriminate between surfaces coated by particles whose size distributions differ by about 5-10 $\mu \mathrm{m}$. Equally important, this discrimination occurs in the lower end of the predicted size range of the surface fibrillations previously described in osteoarthritic human cartilage. And second, the initial observations on cartilage establish that a qualitative correlation exists between the backscatter amplitude plots and the degree of surface fibrillation at gross intervals from normal to extremely abnormal. These results together suggest that this technique may provide a sensitive means of quantifying and following the cartilage surface changes observed in osteoarthritis.

\section{REFERENCES}

Agemura, D. H.; O'Brien, W. D.; Olerud, J. E.; Chun, L. E.; Eyre, D. E. Ultrasonic propagation properties of articular cartilage at $100 \mathrm{MHz}$. JASA 87:1786-1791; 1990.

Bland, J. H.; Cooper, S. M. Osteoarthritis: A review of the cell biology involved and evidence for reversibility. Management rationally related to known genesis and pathophysiology. Sem. Arthritis Rheum. 14:106-133; 1984.

Brandt, K. D. Seminars in arthritis and rheumatism. Introductory remarks. Sem. Arthritis Rheum. 19,Suppl. 1:1-2; 1990.

De Billy, M.; Cohen-Tenoudji, F.; Jungman, A.; Quentin, G. The possibility of assigning a signature to rough surfaces using ultrasonic backscattering diagrams. IEEE Trans. Sonics Ultrason. SU 23(5):356-363; 1976.

Goodman, J. W. Statistical properties of laser speckle patterns. In: Topics in applied physics. Laser speckle and related phenomena. New York: Springer-Verlag; 1985:9-75.

Grinding Wheel Institute. Specifications for grading abrasive microgrits. American National Standards Institute (ANSI) \#B74.10; 1977.

Mankin, H. J.; Lippiello, L. Biochemical and metabolic abnormalities in articular cartilage from osteo-arthritic human hips. J. Bone Surg. 52A:424-434; 1970.

Minns, R. J.; Steven, F. S.; Hardinge, K. Osteoarthrotic articular cartilage lesions of the femoral head observed in the scanning electron microscopy. J. Pathol. 122:63-70; 1977.

Meyer, C. R.; Chiang, E. A.; Fechner, K. P.; Fitting, D. W.; Williams, D. M.; Buda, A. J. Feasibility of high resolution intravascular ultrasonic imaging catheters. Radiology 168:113-116; 1988a.

Meyer, C. R.; Fitting, D. W.; Chiang, E. H.; Williams, D. M.; Buda, A. J. High resolution intravascular imaging using ultrasonic catheters: Proof of concept. Proc. IEEE 76:1074-1078; 1988b.

Paulus, H. E. Nonsteroidal anti-inflammatory drugs. In: Kelley, W. N.; Harris, E. D.; Ruddy, S.; Sledge, C., eds. Textbook of rheumatology. Philadelphia, PA: W. B. Saunders; 1989:765791.

Quentin, G.; De Billy, M.; Cohen-Tenoudji, F.; Doucet, J.; Jungman, A. Experimental results on the scattering of ultrasound by randomly and periodically rough surfaces in the frequency range 2 to $25 \mathrm{MHz}$. Ultrasonics Symposium Proceedings, IEEE Press, 102-106; 1975.

Serra, J. Introduction to mathematical morphology. Computer vision, graphics, and image processing. 35:283-305; 1986.

Sternberg, S. R. Grayscale morphology. Computer vision, graphics, and image processing. 35:333-355; 1986.

Thorne, P. D.; Pace, N. G. Acoustic studies of broadband scattering from a model rough surface. JASA 75:133-144; 1984.

Zhuang, X.; Haralick, R. M. Morphological structure element decomposition. Computer vision, graphics, and image processing 35:370-382; 1986. 